\title{
Depth rotation and mirror-image reflection reduce affective preference as well as recognition memory for pictures of novel objects
}

\author{
REBECCA LAWSON \\ University of Liverpool, Liverpool, England
}

\begin{abstract}
In two experiments, the identification of novel 3-D objects was worse for depth-rotated and mirrorreflected views, compared with the study view in an implicit affective preference memory task, as well as in an explicit recognition memory task. In Experiment 1, recognition was worse and preference was lower when depth-rotated views of an object were paired with an unstudied object relative to trials when the study view of that object was shown. There was a similar trend for mirror-reflected views. In Experiment 2, the study view of an object was both recognized and preferred above chance when it was paired with either depth-rotated or mirror-reflected views of that object. These results suggest that view-sensitive representations of objects mediate performance in implicit, as well as explicit, memory tasks. The findings do not support the claim that separate episodic and structural description representations underlie performance in implicit and explicit memory tasks, respectively.
\end{abstract}

Memory has been examined in study-test experiments with two types of tasks: explicit and implicit. In explicit memory tasks, participants are informed that they are being tested about their memory of the study phase. For example, in the test phase of a recognition memory task, participants must decide whether a stimulus was presented in an earlier study phase. Implicit memory is demonstrated in tasks that do not require people to deliberately recall a study episode (typically no mention is made of the study phase), and yet study does influence their performance. For example, in the test phase of a priming task, participants may have to name some stimuli that they saw at study and some unstudied stimuli. Any difference in performance between the studied and unstudied stimuli suggests that participants had some memory for the study stimuli, even though they may not have been aware that they saw them at study.

A number of researchers have proposed that different types of representations are accessed when participants perform explicit and implicit memory tasks (e.g., Biederman \& Cooper, 1991, 1992; Biederman \& Gerhardstein, 1993; Cooper, Schacter, Ballesteros, \& Moore, 1992; Schacter, Cooper, \& Delaney, 1990; Schacter, Cooper, Delaney, Peterson, \& Tharan, 1991; Seamon, et al., 1997). For example, episodic representations may mediate explicit memory tasks while structural description representations may be used in implicit memory tasks, with these two types of representations having different qualities.

I gratefully acknowledge the support of the Leverhulme Trust and Heinrich Bülthoff at the Max-Planck-Institut für Biologische Kybernetik. Correspondence should be addressed to R. Lawson, Department of Psychology, University of Liverpool, Eleanor Rathbone Building, Bedford Street South, Liverpool L69 7ZA, England (e-mail: rlawson@ liverpool.ac.uk).
Of particular relevance to the present article, episodic representations have been assumed to encode view-specific information about the study stimulus such as its size, view in depth and left-right orientation (i.e., its mirror-image version). This theoretical claim is based on reports that, for explicit memory measures such as recognition memory, performance is worse if there are study-test perceptual changes in the stimuli presented, such as its size (e.g., Biederman \& Cooper, 1992; Cooper et al., 1992; Jolicoeur, 1987; Milliken \& Jolicoeur, 1992; Seamon et al., 1997), its view in depth (e.g., Humphrey \& Khan, 1992; Seamon \& Delgado, 1999; Srinivas \& Verfaellie, 2000), and its mirror-image version (Biederman \& Cooper, 1991; Cooper et al., 1992; Seamon et al., 1997).

In contrast, structural descriptions have been assumed to be relatively abstract and view-invariant representations coding the parts and spatial relations between the parts of the object but not metric details of the stimulus (Biederman \& Cooper, 1991, 1992). Test stimuli should be matched equally effectively to such representations whether or not the study and test stimuli have the same size, view in depth or mirror-image version, so long as the same parts in the same spatial relations are visible in both study and test stimuli (Biederman \& Gerhardstein, 1993). This account is consistent with the finding that in implicit memory tasks such as priming, performance has been reported to be invariant to study-test changes in size (Biederman \& Cooper, 1992; Cooper et al., 1992; Seamon et al., 1997), rotation in depth (Biederman \& Gerhardstein, 1993; Seamon \& Delgado, 1999; Srinivas $\&$ Verfaellie, 2000), and mirror-image reflection (Biederman \& Cooper, 1991; Fiser \& Biederman, 2001; Seamon et al., 1997). Furthermore, Biederman and colleagues have argued that these structural descriptions 
(rather than the episodic representations) subserve everyday object recognition.

However, contrary to the null effects reported above, there is evidence that the representations accessed in implicit memory tasks are also view sensitive. For example, although Biederman and Gerhardstein (1993) reported view-invariant priming across depth rotations with a picture-naming task, Lawson and Humphreys (1998) found clear view sensitivity with the same task and similar stimuli. They reported greater priming of pictures of familiar objects when the view in depth of the object was the same at study and test as when a different, depthrotated view was presented at test (see also Srinivas, 1993, 1995). Similarly, Srinivas (1996) found size-sensitive priming effects for pictures of familiar objects (cf. Biederman \& Cooper, 1992).

Seamon and colleagues noted that almost all implicit memory tasks tested to date had measured priming (Seamon \& Delgado, 1999; Seamon et al., 1997). They suggested that alternative tasks should be used to provide converging evidence about the view sensitivity of the representations mediating performance in implicit memory. In their experiments, they used affective preference and recognition memory as measures of implicit and explicit memory, respectively. The affective preference task exploits the mere exposure effect. This is the increase in people's preference at test for novel stimuli that were experienced but not reinforced in a prior study phase ( $\mathrm{Za}-$ jonc, 1968). The mere exposure effect can occur for stimuli seen so briefly at study that subsequent recognition memory for the stimuli is at chance (e.g., Kunst-Wilson \& Zajonc, 1980; Newell \& Bright, 2003). It has been argued that the mere exposure effect reflects implicit memory processes (Schacter, 1987; Seamon, et al., 1997; Seamon et al., 1995; Squire, 1992; but see Whittlesea \& Price, 2001). For example, Seamon et al. (1995, p. 719) argued that in their experiments, participants did not intentionally or voluntarily try to retrieve information about the study items when making their preference judgments.

In support of the claim outlined above that viewsensitive episodic representations are tapped by explicit memory tasks while relatively view-invariant structural descriptions are tapped by implicit memory tasks, Seamon et al. (1997) reported evidence for view sensitivity on recognition tasks but not on affective preference tasks. Seamon et al. (1997) presented pictures of novel objects at study in an incidental learning task. At test, participants were given a two-alternative forced choice and were asked to either recognize which stimulus had been presented at study or which stimulus they preferred. These experiments indirectly measured view sensitivity by presenting same and different views of the study stimuli on separate trials. For both trial types, the distractor was a view of an unstudied object. Seamon et al. (1997) found that study-test changes in mirrorimage reflection and size impaired recognition memory but had no effect on affective preference. They suggested that perceptual information (such as size and mirror- image version) is coded in the episodic representations tapped by recognition memory tasks, but it is not coded in the structural descriptions accessed in affective preference tasks.

In similar experiments, Seamon and Delgado (1999) showed participants pictures of novel objects during incidental learning at study. As in Seamon et al. (1997), their first experiment presented same and different views of the study object on separate trials to indirectly measure view sensitivity. They found that study-test changes produced by an $80^{\circ}$ depth rotation impaired recognition memory but not affective preference. Their second experiment replicated the first, except that at test both pictures showed the same studied object. The target depicted the studied object in the studied view while the distractor depicted the studied object in an $80^{\circ}$ depthrotated view. This experiment therefore directly measured view sensitivity. Participants were above chance at recognizing the studied view, but their preference for the studied view was at chance. Seamon and Delgado (1999) argued that their results provided further support for Biederman and Cooper's $(1991,1992)$ claims that the episodic representations tapped by explicit memory tasks are view sensitive, whereas the structural descriptions tapped by implicit memory tasks are relatively view invariant. In their first experiment, participants were better at recognizing objects depicted from the same view compared with a different view from that seen at study, and in their second experiment participants could recognize which of two views in depth of an object they had seen at study. Both results suggest that view in depth was encoded in episodic representations. In contrast, in their first experiment there was no increase in preference for the study object if it was seen at the same view relative to a different view in depth, and in their second experiment participants did not prefer the same view over an unstudied view in depth of the study object. Both results suggest that view in depth was not encoded in structural descriptions.

However, caution may be needed in interpreting the results of Seamon and Delgado (1999). First, there was probably more power to detect differences in performance in the recognition memory task relative to the affective preference task. Second, the preference results showed a trend in the same direction as the recognition memory results. Third, the depth rotation tested by Seamon and Delgado was confounded with a near mirrorimage reflection. Fourth, there is disagreement as to the theoretical interpretation of dissociations in performance across different memory tasks.

Taking the first two issues together, recognition memory on same-view trials was $88.1 \%$ in Seamon and Delgado's (1999) first experiment, whereas preference on same-view trials was only $63.1 \%$. This relatively weak preference may have made it difficult to detect a further drop in preference on different-view trials, and there was a trend in this direction, with only $57.5 \%$ correct. Although in absolute terms, this $5.6 \%$ reduction in preference on different-view trials was less than the corresponding $10.6 \%$ reduction in 
recognition memory, it represents a greater proportional change in performance relative to chance $(50 \%)$. Similar concerns can be raised about their second experiment. Here, recognition memory on same-view trials was just $64.2 \%$, so this was a difficult task, and again there was a trend for above chance performance on the preference task, with $51.6 \%$ correct.

Such concerns are not specific to Seamon and Delgado's (1999) experiments. Instead, it may be easier to detect view sensitivity in explicit memory tasks because these tasks are often more reliable than implicit memory tasks (Buchner \& Brandt, 2003; Buchner \& Wippich, 2000; Meier \& Perrig, 2000). The reliability of a measure directly influences statistical power in such a way that the use of less reliable measures will reduce the likelihood of finding significant effects of an independent variable (Hallahan \& Rosenthal, 1996; Meier \& Perrig, 2000). For example, Buchner and Brandt (2003) compared performance on an explicit recognition task and an implicit preference task. Participants first studied line drawings of objects in an incidental learning task. Different groups of participants then decided which pictures they either had seen at study (in the explicit memory task) or preferred (in the implicit memory task). In two experiments, they found that performance was much more reliable on the explicit memory task than on the implicit memory task, although preference was above chance in both experiments. This may be because the correct response in explicit memory tasks is usually well specified (e.g., "identify the old item"), whereas participants often have considerable freedom in deciding how to perform an implicit memory task (e.g., "which item do you prefer?").

The reduced reliability of measures of implicit memory in comparison with explicit memory may have been an artifact that contributed to the many dissociations reviewed above that reported view sensitivity on explicit memory tasks and view invariance on implicit memory tasks. Furthermore, if implicit memory tasks tend to be unreliable, this could also explain discrepancies in the literature regarding the view sensitivity of implicit memory tasks. View sensitivity has been reported on some occasions (e.g., Lawson \& Humphreys, 1998; Srinivas, 1993, 1995, for depth rotation; Stankiewicz, Hummel, \& Cooper, 1998, for mirror-image reversal) but not on others (e.g., Biederman \& Gerhardstein, 1993, for depth rotation; Biederman \& Cooper, 1991; Fiser \& Biederman, 2001; Stankiewicz et al., 1998, for mirror-image reversal). Even when significant effects of view sensitivity have not been found in implicit memory tasks, a trend toward view sensitivity has often been reported, as was the case in Seamon and Delgado's (1999) experiments. This is again consistent with the finding that implicit memory tasks are often relatively unreliable.

Regarding the third issue, the $80^{\circ}$ depth rotation used by Seamon and Delgado (1999) happened to produce a near mirror-image reflection, as Seamon and Delgado themselves noted (see their Figure 1 and pp. 150, 159160). Seamon et al. (1997) found that a mirror-image re- flection had no influence on preference. If the particular depth rotation tested by Seamon and Delgado did not influence preference because it was a near mirror-image reflection, other depth rotations may affect preference. The present experiments re-examined the claims of Seamon and Delgado but with stimuli on different-view trials that were either depth-rotated views or mirror-image reflections of the study stimuli, so that the effects of these two manipulations could be examined separately.

Finally, on the fourth issue, numerous dissociations have been found between performance on explicit and implicit memory tasks for a variety of perceptual manipulations, a number of which have been detailed above. However, there have also been reports of dissociations between performance on different implicit memory tasks. For example, in their second experiment, Vernon and Lloyd-Jones (2003) reported color-sensitive priming when the implicit memory test task was coloredobject decision but not when it was object naming. Only the test task differed between the two implicit memory groups, with both groups being shown identical stimuli and being given the same study tasks. Also, Fiser and Biederman (2001) reported translation-invariant priming, while Bar and Biederman $(1998,1999)$ found translationsensitive priming. Fiser and Biederman suggested that this dissociation between performance on two implicit memory tasks "might indicate that the dominant representations that are responsible for priming in the two cases (although both visual) are different" (p. 232). However, the conclusion that there are potentially many different types of representations subserving implicit memory (in addition to separate representations that are accessed in explicit memory tasks) seems unparsimonious. Alternative explanations may be more appealing. Liu and Cooper (2001) ran three object priming experiments. Each used similar study and test stimuli and the same study task (left-right direction of facing) but tested implicit memory with different tasks: im/possible object decision, symmetry judgment, and picture-picture matching. They found a different pattern of priming in the $\mathrm{im} /$ possible object decision task relative to the latter two tasks and argued that such dissociations should not be assumed to indicate that different, stored object representations are being accessed. Instead, they suggested that, since performance dissociated across different tasks, all of which supposedly tapped implicit memory, it seems likely that such dissociations reflect differences in the tasks' processing demands.

These examples of task-dependent dissociations in measures of implicit memory call into question the theoretical interpretation that dissociations in performance across explicit and implicit memory tasks imply that these tasks also tap different representations. As noted above, it seems more appealing to interpret dissociations in performance between different implicit memory tasks in terms of task-dependent processing demands. Likewise, dissociations in performance across implicit and explicit memory tasks may be best accounted for in 


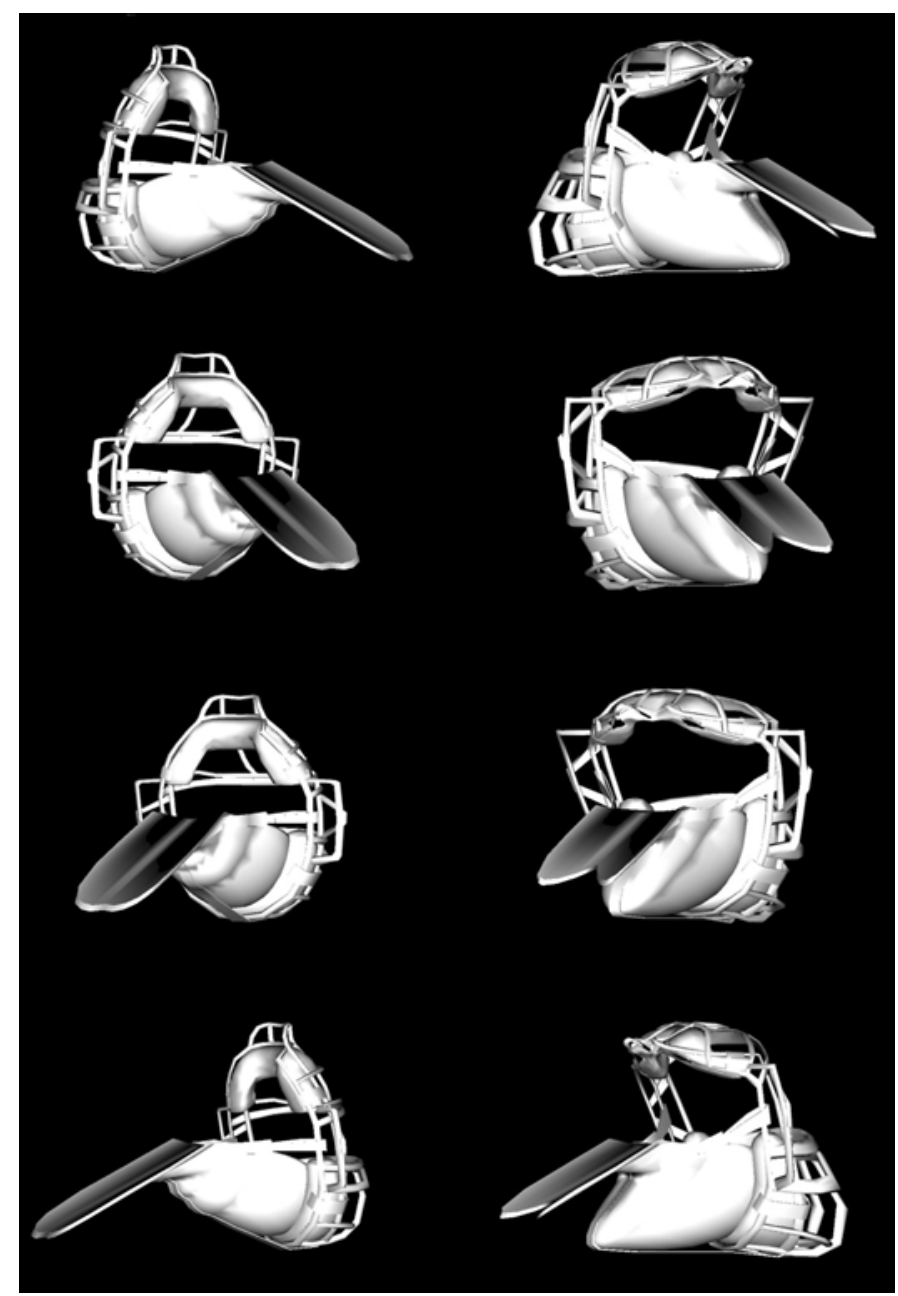

Figure 1. Examples of the eight different views of 1 of the 24 objects used in Experiments 1 and 2. The left column shows four views of one morph, and the right column shows four views of the other morph. In the first and second rows, right-facing views are depicted at $60^{\circ}$ and $30^{\circ}$ rotations from the foreshortened $0^{\circ}$ view, respectively. In the third and fourth rows, the views depict (left-facing) mirror-image reflections of the views in the second and first rows, respectively.

terms of different processing strategies, rather than distinct episodic and structural description representations. Explicit and implicit memory tasks may typically lead to the adoption of distinct processing strategies and hence to the retrieval of different types of information from the same, stored object representations (Whittlesea \& Price, 2001). Whittlesea and Price found that alternative processing strategies could be encouraged in both explicit and implicit memory tasks and that this resulted in "implicit-like" performance on explicit memory tasks and vice versa. They argued that there is just one memory system, which is used in different ways in different tasks. Tarr and colleagues (Tarr, 2003; Tarr \& Cheng, 2003), arguing from different sets of results, have reached rather similar conclusions. They did not assume that there are multiple, independent sets of stored object representations. Instead, they proposed that object identification for different stimuli in different tasks requiring either specific or more general levels of categorization for both novice and expert participants may be supported by the same, flexible system for visual object recognition.

In summary, Seamon and colleagues have argued that their results revealing the view sensitivity of recognition (a measure of explicit memory) and the view invariance of affective preference (a measure of implicit memory) support the claims of Biederman and Cooper $(1991,1992)$ in indicating that distinct representations mediate performance in explicit and implicit memory tasks (Seamon \& Delgado, 1999; Seamon et al., 1997). There are, however, alternative reasons why view-sensitive performance might be found in explicit memory tasks and view-invariant performance in implicit memory tasks. First, it may be easier 
to detect view sensitivity in explicit memory tasks than in implicit memory tasks since explicit memory tasks are usually more reliable and more powerful (Buchner \& Brandt, 2003; Meier \& Perrig, 2000). Second, explicit and implicit memory tasks may encourage participants to adopt different processing strategies (Whittlesea \& Price, 2001 ) that may result in their being differentially sensitive to viewpoint.

The two experiments reported here replicated the design of Seamon and colleagues' experiments (Seamon \& Delgado, 1999; Seamon et al., 1997). The experiments tested whether, for explicit memory (recognition) tasks and implicit memory (preference) tasks, performance is influenced by study-test changes in mirror-image reflection and view in depth. As in Seamon and Delgado's first experiment, Experiment 1 here tested view sensitivity indirectly. On same-view trials, the studied view of a studied object was presented together with a view of an unstudied object. On different-view trials, an unstudied view of a studied object was presented with a view of an unstudied object. If the studied object is selected more on same-view than on different-view trials, performance is view sensitive. As in Seamon and Delgado's second experiment, Experiment 2 here tested view sensitivity directly. Here, studied and unstudied views of a study object were presented simultaneously. If the studied view is selected more than the unstudied view, performance is view sensitive.

Performance in the present experiments was sensitive to depth rotation and to mirror-image reflection on both explicit and implicit memory tasks, and there was a similar pattern of results for both tasks. This failed to replicate Seamon and colleagues' (Seamon \& Delgado, 1999; Seamon et al., 1997) findings of a qualitative difference in the view sensitivity of explicit and implicit measures of memory. The present results are not consistent with the claim that relatively view-invariant structural descriptions are accessed in implicit memory tasks (Biederman \& Cooper, 1991, 1992; Fiser \& Biederman, 2001). Instead, the findings are compatible with the proposal by Tarr and colleagues (Tarr, 2003; Tarr \& Cheng, 2003) that a single set of view-sensitive, stored representations may underlie visual object recognition across a range of tasks and types of stimuli.

\section{EXPERIMENT 1}

Experiment 1 replicated and extended the design of the first experiment of Seamon and Delgado (1999), in which participants had to detect shape changes while ignoring view changes. Participants first studied 24 views of morphs of novel objects, repeatedly rating the complexity of each stimulus. After this incidental learning study task, they received one of two surprise test tasks: a recognition memory task or an affective preference task. Trials for both test tasks presented a target and a distractor picture simultaneously. In the recognition memory task, participants were asked to choose the stimulus that they had seen at study. In the affective preference task, participants were told to choose the stimulus that they preferred.

On each test trial, the target depicted a view of the study object, and the distractor depicted a morphed version of the study object (see Figure 1). There were three types of test trials (see Figure 2). Different morph (MORPH) trials presented the target at the same view as that at which the morph had been seen during study; that is, the study and target stimuli were identical. Mirror-image (MIRROR) trials presented the target mirror-reflected from the view of the morph that had been seen at study. Depth-rotated (DEPTH) trials presented the target rotated $30^{\circ}$ in depth from the view of the morph that had been seen at study. In each of these three conditions, the distractor was depicted from the same view as the target, but it showed a different morph of the object than had been presented at study (see Figure 2).

The MORPH trials were equivalent to the same-view trials tested by Seamon and Delgado (1999). The DEPTH and MIRROR trials were like the different-view trials performed by Seamon and Delgado in their first experiment except that the $80^{\circ}$ depth-rotated views tested by Seamon and Delgado were also nearly mirror-image reflections of each other. In the present experiment, this confound was removed by examining the effects of depth rotation and mirror-image reflection separately, on DEPTH and MIRROR trials, respectively.

\section{Method}

\section{Participants}

Forty-eight undergraduate students from the University of Liverpool volunteered to take part in the experiment for course credit. No participants were replaced.

\section{Materials}

A set of pictures of morphs of 24 novel objects was used (see Lawson, Bülthoff, \& Dumbell [2003] for further details). Each object was represented by two morphs (S3 and S11 on Lawson et al.'s coding). Each morph was depicted from two views in depth, $30^{\circ}$ and $60^{\circ}$. Each of these views could, in turn, be presented in two mirror-image versions. In total, there were thus eight depictions of each of the $24 \mathrm{ob}-$ jects (two morphs $\times$ four views; see Figure 1). The (unseen) $0^{\circ}$ view was assigned to be a foreshortened view if the object had an elongated shape. Otherwise, it was arbitrarily assigned. Not all of the objects were bilaterally symmetrical, although most were nearly so. Hence, the mirror-image reflections were usually very similar but not identical to either a $60^{\circ}$ or a $120^{\circ}$ depth rotation.

\section{Design}

Each participant completed three study blocks followed by either a recognition memory test block or an affective preference test block. There were 24 trials per block, with every object being seen once per block. Across the three study blocks, the same picture of a morph of a given object was shown three times to a given participant. Each participant saw a subset of three objects at each of the eight possible depictions of the object (two morphs $\times$ four views for each object). Eight study files were produced by rotating the eight subsets of three objects in a Latin square design across the eight possible depictions of each object. In the recognition memory and affective preference test trials, pictures of the target and distractor were presented simultaneously. On all test trials, the target 

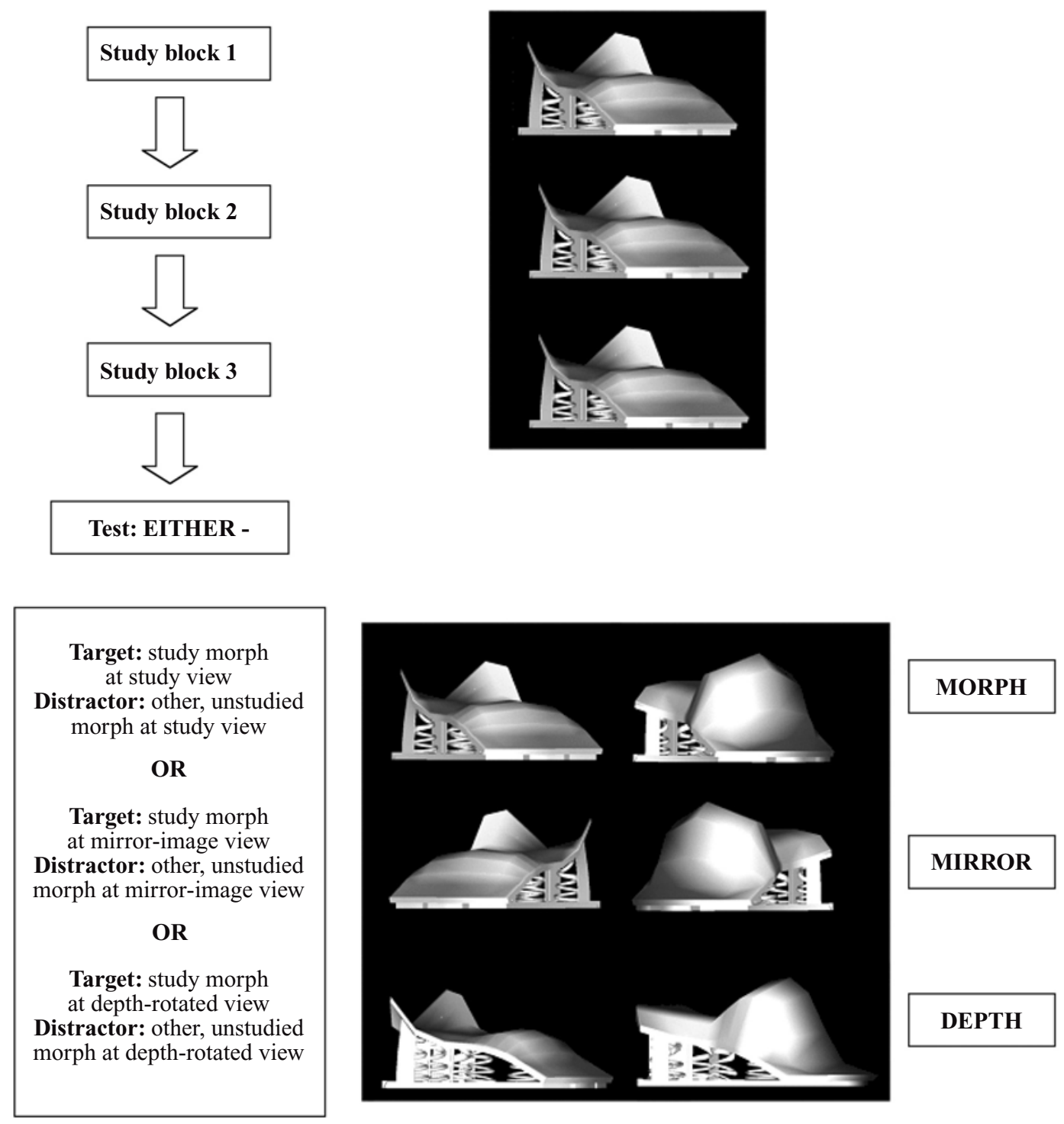

Figure 2. Stimuli and design for Experiment 1, which indirectly measured view sensitivity to mirror-image reflection and to depth rotation. The task in the three study blocks was to rate the complexity of the picture (a $30^{\circ}$ right-facing view is depicted here). At test, participants had to decide which of the two objects had been presented at study (in the recognition memory task) or which of the two objects they liked more (in the affective preference task). The target is depicted on the left and the distractor on the right for the three types of test trials (MORPH, MIR$\mathrm{ROR}$, and DEPTH). Here, the target and distractor both depict a $30^{\circ}$ right-facing view, a $30^{\circ}$ left-facing view, and a $60^{\circ}$ right-facing view in the MORPH, MIRROR, and DEPTH trials, respectively.

showed the morph of the object seen at study, and the distractor was depicted from the same view as that of the target, but it showed a different morph of the object than that seen at study. There were equal numbers of three types of test trials (see Figure 2). Different morph (MORPH) trials showed the target at the same view as that at which it had been seen during study, so the study and target stimuli were identical. Mirror-image (MIRROR) trials presented the target mirror reflected from the view at which it had been seen at study. Depth-rotated (DEPTH) trials presented the target rotated $30^{\circ}$ in depth from the view at which it had been seen at study. There were three different test files. In each test file, four objects were seen in each of six conditions (on MORPH, MIRROR, or DEPTH trials, with the target presented to the left or to the right of fixation). Six participants were assigned to each of the eight study files; 2 of these were assigned to each of the three test files, of whom 1 did the recognition memory test block, and the other did the affective preference test block. Trials were presented in a different random order for each participant in every block.

\section{Apparatus and Procedure}

At study, participants were instructed that the experiment was intended to investigate perceptual decision making and that they had to rate the complexity of the pictures on a scale of 1 to 9 . Each picture was presented for $2,500 \mathrm{msec}$. The next picture was presented $1,000 \mathrm{msec}$ after they had responded. They were given a self-paced break between each study block. In the recognition memory test task, the participants had to decide which of the two depicted objects they had seen during the complexity rating study task. They 
were told to guess if they could not remember which object they had seen. In the affective preference test task, the participants had to decide which of the two presented objects they preferred. They were told to respond even if they had no strong preference for either object. In both the recognition memory and the affective preference tasks, the target and distractor pictures were presented simultaneously until the participants responded by pressing the $M$ key (for the right picture) or the $\mathrm{Z}$ key (for the left picture). The next picture was presented $1,000 \mathrm{msec}$ after they had responded; the participants were not encouraged to respond rapidly.

\section{Results}

Analyses of variance (ANOVAs) were conducted on the mean percentage correct in the recognition memory and affective preference tasks (see Figure 3). The results for the $F$ values and $t$ values in the by-participants and by-items analyses are reported using subscripts $F_{\mathrm{p}}, F_{\mathrm{i}}, t_{\mathrm{p}}, t_{1}$, respectively. In all analyses, there was one within-participants factor, distractor, which specified how the distractor differed from the study view of that object (MORPH, MIRROR, or DEPTH).

\section{Recognition test task}

Distractor was significant $\left[F_{\mathrm{p}}(2,46)=11.038, p<\right.$ $\left..001 ; F_{\mathrm{i}}(2,46)=16.744, p<.001\right]$. In two-tailed planned comparisons, DEPTH trials $(69.3 \%$ correct $)$ were harder than MORPH trials $(89.6 \%)\left[t_{\mathrm{p}}(23)=23.653, p<.001\right.$; $\left.t_{\mathrm{i}}(23)=30.606, p<.001\right]$. In addition, MIRROR trials $(82.8 \%)$ were harder than MORPH trials $(89.6 \%)\left[t_{\mathrm{p}}(23)=\right.$ $\left.3.524, p<.08 ; t_{\mathrm{i}}(23)=6.760, p<.02\right]$. In one-tailed $t$ tests, performance was above chance $(50 \%)$ in all three conditions $\left[t_{\mathrm{p}}(23)\right.$ and $t_{\mathrm{i}}(23)>5.0$ and $\left.p<.001\right]$.

\section{Preference test task}

There was a marginally significant effect of distractor $\left[F_{\mathrm{p}}(2,46)=2.730, p<.08 ; F_{\mathrm{i}}(2,46)=3.197, p<.06\right]$.
In two-tailed planned comparisons, DEPTH trials (55.7\% studied targets preferred) were harder than MORPH trials $(66.7 \%)\left[t_{\mathrm{p}}(23)=4.374, p<.05 ; t_{\mathrm{i}}(23)=5.375, p<\right.$ $.03]$. In contrast, although the trend was the same as for the recognition task, MIRROR trials $(60.9 \%)$ were not significantly harder than MORPH trials $(66.7 \%)\left[t_{\mathrm{p}}(23)=\right.$ $\left.2.638, p<.2 ; t_{\mathrm{i}}(23)=2.149, p<.2\right]$. In one-tailed $t$ tests, performance was above chance $(50 \%)$ in all three conditions $\left[t_{\mathrm{p}}(23)\right.$ and $t_{\mathrm{i}}(23)>4.2, p<.001$ for MORPH trials; $>2.8, p<.005$ for MIRROR trials; and $>1.7, p<$ .05 for DEPTH trials].

\section{Discussion}

In Experiment 1, performance was view sensitive in an implicit memory task testing affective preference as well as in an explicit recognition memory task. Participants both preferred and recognized same-view targets (on MORPH trials) more than different-view targets (on DEPTH and MIRROR trials), although the trend was not reliable for mirror-reflected targets. The difference between preference on MORPH and DEPTH trials failed to replicate Seamon and Delgado's (1999) first experiment, which found that only recognition, and not preference, was sensitive to depth rotation of the study object. There are a number of reasons why the present experiment may have been more sensitive to viewpoint effects than Seamon and Delgado's first experiment. In the present experiment, there were $20 \%$ more participants, who were tested on $50 \%$ more study items, which were shown three times, rather than just twice. In addition, the study task used here (rating complexity) may have promoted more perceptual processing than the relatively superficial (left-right facing) task used by Seamon and Delgado.

The present finding is not consistent with the claims of Biederman and Cooper (1992) and Seamon and col-

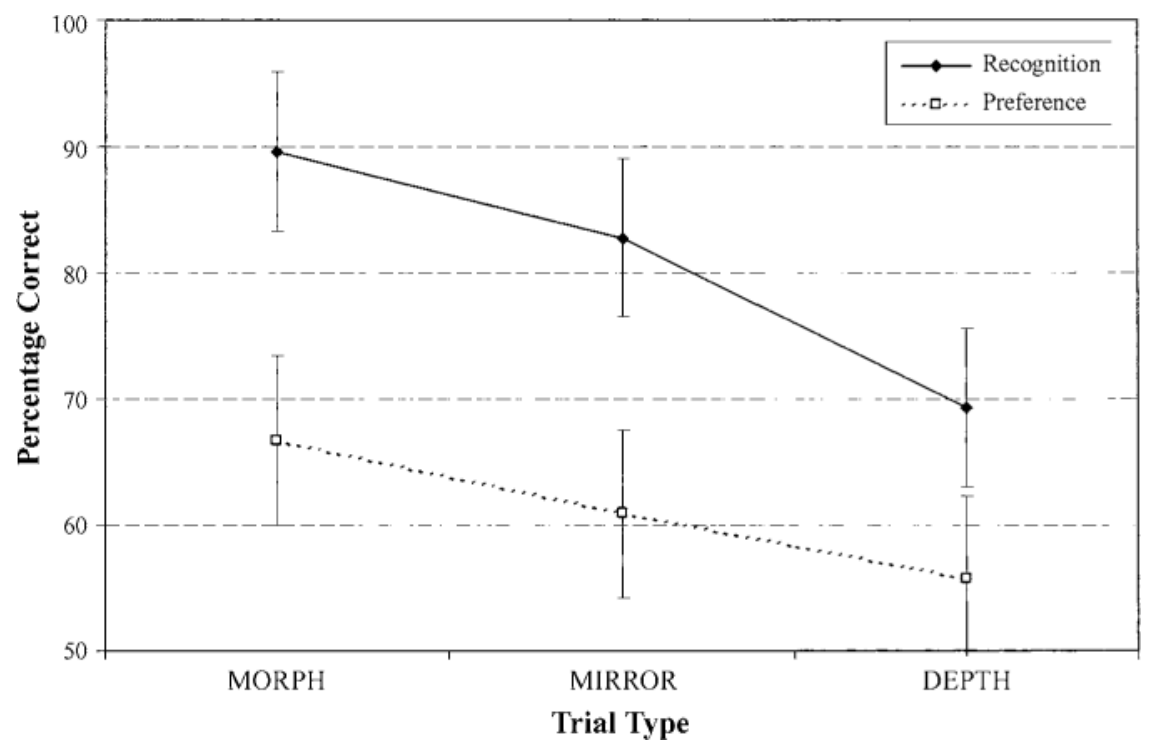

Figure 3. Results for Experiment 1: Percentage correct on MORPH, MIRROR, and DEPTH trials in the recognition memory task and the affective preference task, plotted with $95 \%$ confidence intervals. 
leagues (Seamon \& Delgado, 1999; Seamon et al., 1997) that the structural description of an object accessed in implicit memory tasks such as priming and preference is relatively view invariant. Instead, the difference in preference between MORPH and DEPTH trials replicates the finding of reduced priming on trials presenting depthrotated targets relative to same-view targets (Lawson \& Humphreys, 1998; Srinivas, 1993, 1995). This result provides support for accounts of object recognition that propose that multiple, view-specific object representations are stored (e.g., Tarr, 1995, 2003; Tarr \& Cheng, 2003). Different depth-rotated views of an object may optimally activate different representations leading to view-sensitive performance in implicit memory tasks such as priming and preference, as well as in explicit recognition memory tasks.

The unexpected trend toward a difference in preference between MORPH and MIRROR trials was examined further in Experiment 2. Measures of explicit memory are usually sensitive to the mirror-image version of the stimulus presented (e.g., Biederman \& Cooper, 1991; Cooper et al, 1992; Seamon et al., 1997; Srinivas, 1996, when directly although not when indirectly testing sensitivity to mirror-image version). This result was replicated here. In an implicit memory task, Seamon et al. (1997) found that preference was insensitive to mirrorimage reflection. However, in an alternative implicit memory task-priming-performance has been found to be sensitive to mirror-image reflection. In a picturenaming task, Stankiewicz et al. (1998) found increased priming when familiar objects were primed by the same view compared with a mirror-image reflected picture of an object over priming intervals of a few seconds. Similarly, Henderson and Siefert (1999) reported greater transsaccadic priming by identical views relative to mirrorimage views of familiar objects (see also Pollatsek, Rayner, $\&$ Collins, 1984). For priming intervals of a few minutes, there have been several reports of mirror-image invariant priming for the identification of pictures of familiar objects (Biederman \& Cooper, 1991; Fiser \& Biederman, 2001; Srinivas, 1996; Stankiewicz et al., 1998), although Cooper et al. (1992) found increased priming to sameview compared with mirror-image reflected views of possible objects in an implicit object decision task, but this difference was not reliable.

Thus, over short durations of several seconds, implicit memory has been found to be reduced, but not eliminated, for mirror images relative to the same view of a study stimulus (Henderson \& Siefert, 1999; Pollatsek et al., 1984; Stankiewicz et al., 1998). Over longer durations of a few minutes, measures of implicit memory may be invariant to mirror-image reflection (Biederman \& Cooper, 1991; Fiser \& Biederman, 2001; Seamon et al., 1997; Srinivas, 1996; Stankiewicz et al, 1998) even though explicit recognition is still sensitive to mirrorimage reflection. However, as discussed in the introduction, measures of implicit memory may be less reliable than measures of explicit memory (Buchner \& Brandt,
2003; Buchner \& Wippich, 2000; Meier \& Perrig, 2000). This would reduce the power to detect sensitivity to mirrorimage reflection in implicit memory tasks and, for example, in their second experiment Cooper et al. (1992) found a trend toward priming for possible objects that was sensitive to mirror-image reflection which persisted over several minutes. Experiment 2 investigated this issue by directly testing the sensitivity of a different implicit memory task - preference-to mirror-image reflection using a relatively long study-test duration of several minutes with a reasonably large number of participants.

\section{EXPERIMENT 2}

Experiment 2 attempted to replicate and extend Seamon and Delgado's (1999) second experiment in which participants had to detect view changes. As in Experiment 1 , the participants first completed an incidental learning task during the study period. This was followed by a surprise test task: either recognition memory or affective preference (see Figure 4). Unlike in Experiment 1, MIRROR trials now presented two stimuli that were identical, except for a mirror-image reflection (see Figure 4). DEPTH trials presented two stimuli that were identical, except for a $30^{\circ}$ rotation in depth. MoRPH trials presented two stimuli that were identical, except for a change in shape. For MIRROR and DEPTH trials, the stimulus seen as a target in those trials in Experiment 1 became the distractor in Experiment 2. The MORPH trials in Experiments 1 and 2 were identical. The MIRROR and DEPTH trials were like the trials tested by Seamon and Delgado in their second experiment, except that the effects of mirrorimage reflection and depth rotation were examined separately on MIRROR and DEPTH trials, respectively.

\section{Method}

\section{Participants}

Ninety-six undergraduate students from the University of Liverpool volunteered to take part in the experiment for course credit. No participants were replaced.

\section{Materials}

The stimuli were the same as those in Experiment 1.

\section{Design}

The design was identical to that of Experiment 1 except for the following points. First, there were four, not three, study blocks to try to increase learning during the study phase, since Experiment 2 was expected to be somewhat harder than Experiment 1, as was the case in Seamon and Delgado's (1999) second study. Second, 2 participants, not 1 , were assigned to each of the 48 study file, test file, and test task combinations. Third, for MIRROR and DEPTH test trials, what was the target in Experiment 1 was now the distractor in Experiment 2, and the new target was the study view of that same study object (see Figure 4).

\section{Apparatus and Procedure}

This was identical to Experiment 1, except for the instructions given to the participants. In the recognition memory task, the participants were instructed to decide which of the two different pictures shown (rather than which of the two different objects depicted) they had seen in the study task. Similarly, in the affective preference 


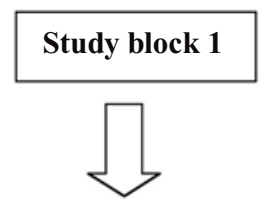

Study block 2

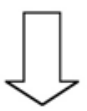

Study block 3

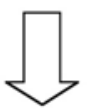

Study block 4<smiles>C1CCCCC1</smiles>

Test: EITHER -

Target: study morph at study view

Distractor: other, unstudied morph at study view

\section{OR}

Target: study morph at study view

Distractor: study morph at mirror-image view

\section{OR}

Target: study morph at study view

Distractor: study morph at depth-rotated view
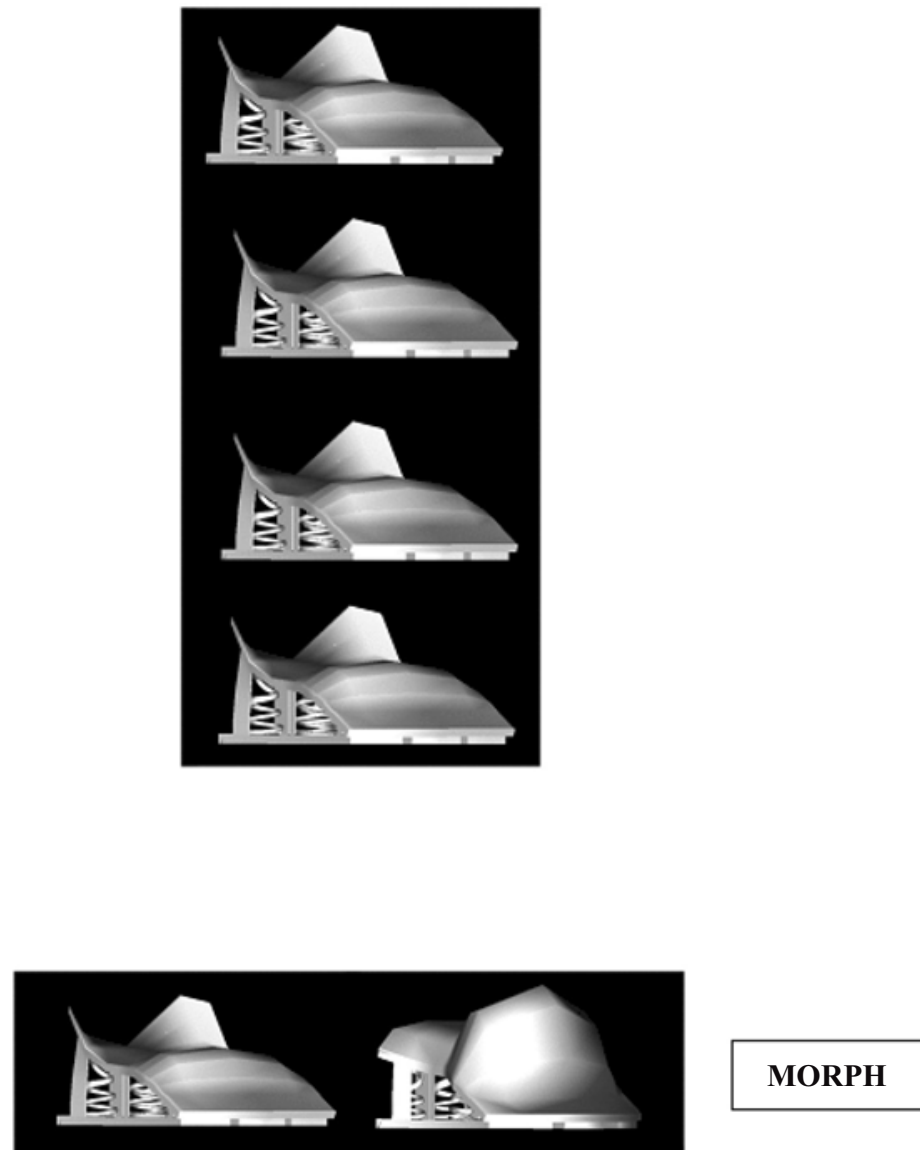

MIRROR
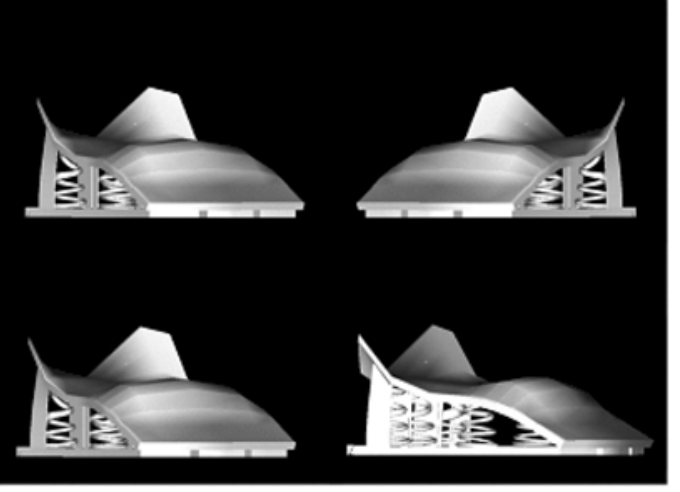

\section{DEPTH}

Figure 4. Stimuli and design for Experiment 2, which directly measured view sensitivity to mirror-image reflection and to depth rotation. The task in the four study blocks was to rate the complexity of the picture (a $30^{\circ}$ right-facing view is depicted here). At test, participants had to either decide which of the two views of the object had been presented at study (the recognition memory task) or which of the two views they liked more (the affective preference task). The target is depicted on the left and the distractor on the right for the three types of test trials (MORPH, MIRROR, and DEPTH). Here, the target always depicts a $30^{\circ}$ right-facing view, and the distractor depicts a $30^{\circ}$ right-facing view, a $30^{\circ}$ left-facing view, and a $60^{\circ}$ right-facing view in the MORPH, MIRROR, and DEPTH trials, respectively.

task, the participants had to decide which of the two pictures shown they preferred.

\section{Results}

ANOVAs were conducted on the mean percentage correct for the recognition memory and preference tasks (see Figure 5). The results for the $F$ values and $t$ values in the by-participants and by-items analyses are reported with subscripts $F_{\mathrm{p}}, F_{\mathrm{i}}, t_{\mathrm{p}}, t_{\mathrm{i}}$, respectively. In all analyses, there was one within-participants factor, distractor, which specified how the distractor differed from the target (MORPH, MIRROR, Or DEPTH). 


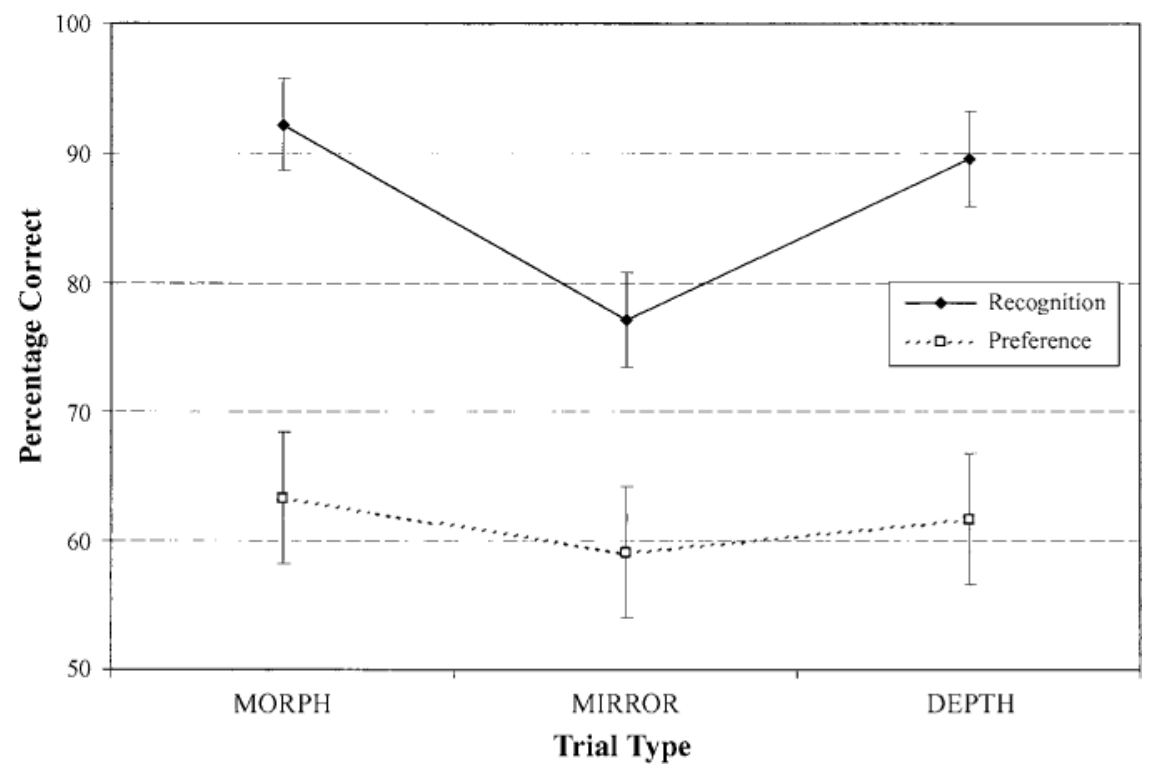

Figure 5. Results for Experiment 2: Percentage correct on MORPH, MIRROR, and DEPTH trials in the recognition memory task and the affective preference task, plotted with $95 \%$ confidence intervals.

\section{Recognition test task}

Distractor was significant $\left[F_{\mathrm{p}}(2,94)=19.759, p<.001\right.$; $\left.F_{\mathrm{i}}(2,46)=17.401, p<.001\right]$. In two-tailed planned comparisons, there was no significant difference between DEPTH trials $(89.6 \%$ correct) and MORPH trials $(92.2 \%)$ $\left[t_{\mathrm{p}}(47)=1.634, p<.3 ; t_{\mathrm{i}}(23)=1.231, p<.3\right]$. However MIRROR trials $(77.1 \%)$ were harder than MORPH trials $(92.2 \%)\left[t_{\mathrm{p}}(47)=27.018, p<.001 ; t_{\mathrm{i}}(23)=27.832, p<\right.$ $.001]$. In one-tailed $t$ tests, performance was above chance $(50 \%)$ in all three conditions $\left[t_{\mathrm{p}}(47)\right.$ and $t_{\mathrm{i}}(23)>7.5$ and $p<.001]$.

\section{Preference test task}

Distractor was not significant $\left[F_{\mathrm{p}}(2,94)=.691, p<.6\right.$; $\left.F_{\mathrm{i}}(2,46)=.764, p<.5\right]$. In two-tailed planned comparisons, there was no significant difference between DEPTH trials $\left(61.7 \%\right.$ correct) and MORPH trials $(63.3 \%)\left[t_{\mathrm{p}}(47)=\right.$ $0.164, p<.7 ; t_{\mathrm{i}}(23)=0.155, \mathrm{p}<.7$ ]. In addition, although the trend was the same as for the recognition task, there was no significant difference between MIRROR trials $(59.1 \%)$ and MORPH trials $(63.3 \%)\left[t_{\mathrm{p}}(47)=1.580, p<\right.$ $\left..3 ; t_{\mathrm{i}}(23)=1.449, p<.3\right]$. Most importantly, in onetailed $t$ tests, performance was above chance $(50 \%)$ in all three conditions $\left[t_{\mathrm{p}}(47)\right.$ and $t_{\mathrm{i}}(23)>3.7$ and $\left.p<.001\right]$.

\section{Discussion}

Replicating the findings in earlier experiments by Seamon and colleagues (Seamon et al., 1997; Seamon \& Delgado, 1999), in Experiment 2 explicit recognition memory was sensitive to both depth rotation and mirrorimage reflection of the studied object. As expected, recognition of the studied view was greater than chance both when it was paired with a depth-rotated view
$(89.6 \%)$ and when it was paired with its mirror image (77.1\%). Similar results were found for the preference task. Experiment 2 therefore failed to replicate the null results reported by Seamon and colleagues for an implicit memory task testing affective preference.

First, Seamon and Delgado (1999) reported that in their second experiment there was no significant preference for studied over depth-rotated views. In contrast, in Experiment 2 here, preference for the studied view over a depthrotated view was significantly greater than chance on DEPTH trials $(61.7 \%)$. As noted in the introduction, one likely reason for this discrepancy was that Seamon and Delgado's second experiment was relatively difficult, as evidenced by the poor performance on the recognition memory task (just $64.2 \%$ correct, compared with $89.6 \%$ correct on DEPTH trials here). In both experiments reported here, as well as in Seamon and Delgado's first experiment, performance was much worse on the preference than on the recognition task. Seamon and Delgado's second experiment therefore probably suffered from a floor effect and had little power to detect a preference for the study view.

Second, Seamon et al., (1997) reported that preference was invariant to mirror-image reflection. However, in Experiment 2 here, preference for the studied view over its mirror image was again significantly greater than chance on MIRROR trials (59.1\%). Note that Seamon et al. tested view sensitivity indirectly by comparing performance on separate same-view test trials and mirrorreflected test trials. This design was analogous to that used in Experiment 1 here (see Figure 2). In contrast, Experiment 2 here tested view sensitivity directly (see Figure 4). It is likely that the latter design provides a more 
powerful measure of view sensitivity. Supporting this, the trend for better performance on same-view (MORPH) trials than on mirror-reflected view (MIRROR) trials was not significant in Experiment 1.

The most important finding from Experiment 2 was that performance was significantly above chance in all conditions for both the recognition and preference tasks. This is discussed further in the General Discussion section. In addition, in the recognition task, MIRROR trials were harder than MORPH trials, whereas there was no significant difference between DEPTH and MORPH trials. Mirror-image reflection thus appears to have less influence on recognition than does depth rotation (see Figure 5). There was no significant difference between MIRROR and MORPH trials in the preference task, but the same trend was found. There was no evidence that either mirrorimage reflection or depth rotation influenced explicit and implicit memory tasks in qualitatively different ways. In both experiments reported here, the same pattern of means was observed across both tasks (see Figures 3 and 5), although the differences between conditions were not always reliable in the implicit memory preference task.

\section{GENERAL DISCUSSION}

The two experiments reported here provide the first evidence that I am aware of for view sensitivity in an implicit memory task other than priming. In both experiments, similar results were found for the explicit recognition memory task and the implicit affective preference task. Experiment 1 indirectly tested view sensitivity. Here, preference (as well as recognition) was greater on trials presenting study views than on trials presenting depthrotated views, with a similar but nonsignificant trend for mirror-reflected views. Experiment 2 directly tested view sensitivity. Here, preference (as well as recognition) was above chance for the study view when it was presented simultaneously with either a depth-rotated view or a mirrorreflected view. These results replicate earlier findings of the view sensitivity of object recognition measured with a different implicit memory task - priming - for depth rotation (e.g., Lawson \& Humphreys, 1998; Srinivas, 1993, 1995 ) and for mirror-image reflection (e.g., Henderson \& Siefert, 1999; Pollatsek et al., 1984; Stankiewicz et al., 1998). The results failed to replicate Seamon and colleagues' reports of a qualitative difference in the view sensitivity of explicit recognition and implicit preference measures of memory to depth rotation and to mirror-image reflection (Seamon \& Delgado, 1999; Seamon et al., 1997).

As discussed in the introduction, a number of experiments have failed to find view-sensitive performance on implicit memory tasks measuring priming (e.g., Biederman \& Gerhardstein, 1993, for depth rotation; Biederman \& Cooper, 1991; Fiser \& Biederman, 2001, for mirrorimage reflection) as well as preference (Seamon \& Delgado, 1999, for depth rotation; Seamon et al., 1997, for mirror-image reflection). I have discussed above several reasons why the present experiments may have detected view sensitivity, whereas in similar experiments Seamon and colleagues have reported null effects. More generally, many of these discrepancies in the literature may be due to the relatively low reliability and power of many measures of implicit memory (Buchner \& Brandt, 2003; Buchner \& Wippich, 2000; Meier \& Perrig, 2000), rather than being evidence of a theoretically important distinction between the representations mediating performance in implicit and explicit memory tasks. In addition, the effect of priming tends to decrease over time, so it is perhaps not surprising to find more reports of view sensitivity for short-term than for long-term priming (e.g., Stankiewicz et al., 1998). Compared with explicit memory tasks, implicit memory tasks may often have little power to detect differences in performance between sameview and different-view trials, even given significant differences between performance on studied and unstudied objects. A further complication with the interpretation of dissociations in performance between explicit and implicit memory tasks is that participants may use different processing strategies in the different tasks. They may therefore retrieve different information even if the same, stored representations are accessed in both tasks (Whittlesea $\&$ Price, 2001).

The present results are inconsistent with claims that relatively view-invariant structural descriptions are accessed in implicit memory tasks, whereas distinct, viewsensitive episodic representations are accessed in explicit memory tasks (Biederman \& Cooper, 1991, 1992; Fiser \& Biederman, 2001; Seamon \& Delgado, 1999). Instead, the results can be accounted for more parsimoniously by assuming that the same view-sensitive representations underlie object recognition across a variety of tasks (including both explicit and implicit memory tasks), with these representations coding both the view in depth and the mirror-image version of stimuli (see also Tarr, 2003; Tarr \& Cheng, 2003).

\section{REFERENCES}

Bar, M., \& Biederman, I. (1998). Subliminal visual priming. Psychological Science, 9, 464-469.

BAR, M., \& BIEDERMAN, I. (1999). Localizing the cortical region mediating visual awareness of object identity. Proceedings of the National Academy of Sciences, 96, 1790-1793.

BIEDERMAN, I., \& COOPER, E. E. (1991). Evidence for complete translational and reflectional invariance in visual object priming. Perception, 20, 585-593.

BIEDERMAN, I., \& COOPER, E. E. (1992). Size invariance in visual object priming. Journal of Experimental Psychology: Learning, Memory, \& Cognition, 13, 640-645.

BiEderman, I., \& GERHARDSTEIN, P. C. (1993). Recognizing depthrotated objects: Evidence for 3-D viewpoint invariance. Journal of Experimental Psychology: Human Perception \& Performance, 19, $1162-1182$.

BuCHNER, A., \& BrandT, M. (2003). Further evidence for systematic reliability differences between explicit and implicit memory tests. Quarterly Journal of Experimental Psychology, 56A, 193-209.

BUCHNER, A., \& WIPPICH, W. (2000). On the reliability of implicit and explicit memory measures. Cognitive Psychology, 40, 227-259.

Cooper, L. A., Schacter, D. L., Ballesteros, S., \& Moore, C. 
(1992). Priming and recognition of transformed three-dimensional objects: Effects of size and reflection. Journal of Experimental Psychology: Learning, Memory, \& Cognition, 18, 43-57.

FISER, J., \& BIEDERMAN, I. (2001). Invariance of long-term visual priming to scale, reflection, translation, and hemisphere. Vision Research, 41, 221-234.

HALlahan, M., \& Rosenthal, R. (1996). Statistical power: Concepts, procedures, and applications. Behavior Research \& Therapy, 34, 489499.

Henderson, J. M., \& Siefert, A. B. C. (1999). The influence of enantiomorphic transformation on transsaccadic object integration. Journal of Experimental Psychology: Human Perception \& Performance, 25, 243-255.

HUMPHREY, G. K., \& Khan, S. C. (1992). Recognising novel views of 3-D objects. Canadian Journal of Psychology, 46, 170-190.

Jolicoeur, P. (1987). A size-congruency effect in memory for visual shape. Memory \& Cognition, 15, 531-543.

Kunst-WILSON, W. R., \& ZAJONC, R. B. (1980). Affective discrimination of stimuli that cannot be recognized. Science, 207, 557-558.

LAwson, R., BülthofF, H. H., \& Dumbell, S. (2003). Interactions between view changes and shape changes in picture-picture matching. Perception, 32, 1465-1498.

LaWSON, R., \& HuMPhreYs, G. W. (1998). View specific effects of depth rotation and foreshortening on the initial recognition and priming of familiar objects. Perception \& Psychophysics, 60, 1052-1066.

LIU, T., \& COOPER, L. A. (2001). The influence of task requirements on priming in object decision and matching. Memory \& Cognition, 29, $\underline{874-882 .}$

MEIER, B., \& PERRIG, W. J. (2000). Low reliability of perceptual priming: Consequences for the interpretation of functional dissociations between explicit and implicit memory. Quarterly Journal of Experimental Psychology, 53A, 211-233.

Milliken, B., \& Jolicoeur, P. (1992). Size effects in visual recognition memory are determined by perceived size. Memory \& Cognition, 20, 83-95.

NEWELL, B. R., \& BRIGHT, J. E. H. (2003). The subliminal mere exposure effect does not generalize to structurally related stimuli. Canadian Journal of Experimental Psychology, 57, 61-68.

PollatseK, A., Rayner, K., \& Collins, W. E. (1984). Integrating pictorial information across eye movements. Journal of Experimental Psychology: General, 113, 426-442.

SCHACTER, D. L. (1987). Implicit memory: History and current status. Journal of Experimental Psychology: Learning, Memory, \& Cognition, 13, 501-518.

Schacter, D. L., Cooper, L. A., \& Delaney, S. M. (1990). Implicit memory for unfamiliar objects depends on access to structural descriptions. Journal of Experimental Psychology: General, 119, 5-24.

Schacter, D. L., CoOper, L. A., Delaney, S. M., Peterson, M. A., \& Tharan, M. (1991). Implicit memory for possible and impossible objects: Constraints on the construction of structural descriptions. Journal of Experimental Psychology: Learning, Memory, \& Cognition, 17, 3-19.

SeAmon, J. G., \& Delgado, M. R. (1999). Recognition memory and af- fective preference for depth-rotated solid objects: Part-based structural descriptions may underlie the mere exposure effect. Visual Cognition, 6, 145-164.

SeAmon, J. G., Ganor-Stern, D., Crowley, M. J., Wilson, S. M., Weber, W. J., O'Rourke, C. M., \& Mahoney, J. K. (1997). A mere exposure effect for transformed three-dimensional objects: Effects of reflection, size, or color changes on affect and recognition. Memory \& Cognition, 25, 367-374.

Seamon, J. G., Williams, P. C., Crowley, M. J., Kim, I. J., Langer, S. A., Orne, P. J., \& Wishengrad, D. L. (1995). The mere exposure effect is based on implicit memory: Effects of stimulus type, encoding conditions, and number of exposures on recognition and affect judgements. Journal of Experimental Psychology: Learning, Memory, \& Cognition, 21, 711-721.

SQUIRE, L. R. (1992). Memory and the hippocampus: A synthesis from findings with rats, monkeys, and humans. Psychological Review, 99. 195-231.

SRINIVAS, K. (1993). Perceptual specificity in nonverbal priming. Journal of Experimental Psychology: Learning, Memory, \& Cognition, 19, $582-602$.

SRINIVAS, K. (1995). Representation of rotated objects in explicit and implicit memory. Journal of Experimental Psychology: Learning, Memory, \& Cognition, 21, 1019-1036.

SRINIVAS, K. (1996). Size and reflection effects in priming: A test of transfer-appropriate processing. Memory \& Cognition, 24, 441-452.

Srinivas, K., \& Verfaellie, M. (2000). Orientation effects in amnesics' recognition memory: Familiarity-based access to object attributes. Journal of Memory \& Language, 43, 274-290.

Stankiewicz, B. J., Hummel, J. E., \& CoOPER, E. E. (1998). The role of attention in priming for left-right reflections of object images: Evidence for a dual representation of object shape. Journal of Experimental Psychology: Human Perception \& Performance, 24, 732-744.

TARR, M. J. (1995). Rotating objects to recognize them: A case study on the role of viewpoint dependency in the recognition of three-dimensional objects. Psychonomic Bulletin \& Review, 2, 55-82.

TARr, M. J. (2003). Visual object recognition: Can a single mechanism suffice? In M. A. Peterson \& G. Rhodes (Eds.), Perception of faces, objects, and scenes: Analytic and holistic processes. Oxford: Oxford University Press.

TARr, M. J., \& Cheng, Y. D. (2003). Learning to see faces and objects. Trends in Cognitive Sciences, 17, 23-30.

Vernon, D., \& Lloyd-Jones, T. J. (2003). The role of colour in implicit and explicit memory performance. Quarterly Journal of Experimental Psychology, 56A, 779-802.

Whittlesea, B. W. A., \& Price, J. R. (2001). Implicit/explicit memory versus analytic/nonanalytic processing: Rethinking the mere exposure effect. Memory \& Cognition, 29, 234-246.

ZaJONC, R. B. (1968). Attitudinal effects of mere exposure. Journal of Personality \& Social Psychology Monographs, 9 (2, Pt. 2), 1-27.

(Manuscript received June 25, 2003; revision accepted for publication February 2, 2004.) 